\title{
Equation of state for partially ionized carbon at high temperatures
}

\author{
Alexander Y. Potekhin: \\ Ioffe Physico-Technical Institute, 194021 St. Petersburg, Russia \\ Gérard Massacriel and Gilles Chabrie1 \\ Ecole Normale Supérieure de Lyon, CRAL (UMR CNRS No. 5574), 69364 Lyon Cedex 07, France
}

(Received 9 December 2004; revised manuscript received 8 August 2005)

\begin{abstract}
Equation of state for partially ionized carbon at temperatures $T \gtrsim 10^{5} \mathrm{~K}$ is calculated in a wide range of densities, using the method of free energy minimization in the framework of the chemical picture of plasmas. The free energy model includes the internal partition functions of bound species. The latter are calculated by a self-consistent treatment of each ionization stage in the plasma environment taking into account pressure ionization. The long-range Coulomb interactions between ions and screening of the ions by free electrons are included using our previously published analytical model.
\end{abstract}

PACS numbers: 52.25.Kn, 05.70.Ce, 52.27.Gr, 64.30.+t

\section{INTRODUCTION}

The understanding of the physical properties of matter at high densities and temperatures is important for the fundamental physics as well as for various physical and astrophysical applications. Since the 1980s the theoretical interest in matter under such unusual conditions (e.g., Refs. 1, 2, 3, 4]) has been enhanced by laboratory developments like high-power short duration lasers, shock-induced plasmas, inertial confinement implosions, or exploding metal wires (e.g., Refs. [5, 6, 7, 8, 9]). In the astrophysical domain the calculation of the equation of state (EOS) for stellar partial ionization zones is a particularly challenging problem. In these zones the electrons and different ionic species cannot be regarded as simple ideal gases: Coulomb interactions, bound-state level shifts, pressure ionization, and electron degeneracy should be taken into account. In this paper, we calculate the EOS for carbon at temperatures $10^{5} \mathrm{~K} \lesssim T \lesssim 10^{7} \mathrm{~K}$ in a wide range of densities $\rho$. Such an EOS is required, e.g., for modeling inner envelopes of carbon-rich white dwarfs [10, 11] or outer envelopes of neutron stars.

An EOS calculation in the partial ionization regime is not possible without approximations. For astrophysical simulations, these approximations should not violate the thermodynamic consistency. The free energy minimization method [12, 13] allows one to include the complex physics in the model and ensures the consistency. This method has the great advantage to identify the various contributions to the free energy, illustrative of various physical effects (see, e.g., Ref. 14], for a discussion).

Free-energy models which carefully include the nonideal effects have been proposed for fluid hydrogen 15. and helium [16, 17]; the EOS tables for these elements,

\footnotetext{
*Electronic address: palex@astro.ioffe.ru

$\dagger$ Electronic address: Gerard.Massacrier@ens-lyon.fr

${ }^{\ddagger}$ Electronic address: chabrier@ens-lyon.fr
}

which cover a pressure and temperature range appropriate for low-mass stars, brown dwarfs, and giant planets have been calculated in [18]. For heavier elements, a similarly detailed EOS is lacking. Up to now, the best available thermodynamically consistent EOS for carbon covering the stellar pressure ionization zones was the EOS developed by Fontaine, Graboske, and Van Horn in the 1970s 14] (FGV) and amended in 1990s [19]. We shall call these two versions FGV77 and FGV99, respectively. This EOS has been calculated by different methods in different $\rho-T$ domains. At relatively low densities (e.g., $\rho<(0.01-1) \mathrm{g} \mathrm{cm}^{-3}$ for $10^{5} \mathrm{~K}$ $<T<10^{6} \mathrm{~K}$ ), the ionization equilibrium has been obtained by the free-energy minimization technique. At densities above several grams per cubic centimeter, the Thomas-Fermi model has been employed. At intermediate densities, in particular in the various regimes of pressure ionization, the EOS was interpolated between these two regions. Clearly, the accuracy of the EOS in the interpolation region can be called into question. Moreover, the Thomas-Fermi model may be inaccurate at $\rho \lesssim 10^{3} \mathrm{~g} \mathrm{~cm}^{-3}$, where the pressure is not sufficiently high to force the complete ionization of carbon, as we shall see below.

Extension of the free energy minimization technique to $\rho \gtrsim 0.1 \mathrm{~g} \mathrm{~cm}^{-3}$ is complicated because of the growing importance of nonideal contributions to the free energy and the onset of pressure ionization. The latter is difficult to treat in the framework of the "chemical picture" of plasmas, which assumes that different ion species can be clearly identified (see, e.g., Refs. 18, 20, 21], for discussion). On the other hand, EOS calculations within the more rigorous "physical picture," quite successful at relatively low $\rho$ (e.g., [22]), become prohibitively complicated at such high densities. First principle approaches based on path integral Monte Carlo (PIMC) 23] or molecular dynamics (MD) calculations are computationnaly highly expensive. These methods also suffer from some difficulties. Indeed, the sign or node problem for the PIMC method or the use of effective pair potentials for MD 
simulations restrict their applicability (see however [2]). In any case, a comparison with our results will be instructive, but, to the best of our knowledge, no PIMC or MD data for carbon in the temperature-density range of interest in this paper has been published yet.

In this paper we present an EOS model which relies on the free energy minimization in the framework of the chemical picture and extends to arbitrarily high densities across the pressure ionization region without interpolation. This allows us to obtain not only the thermodynamic functions, but also number fractions for every ionization stage. We treat the long-range interactions in the system of charged particles (ions and electrons) using the theory previously developed for fully ionized plasmas 25, 26]. The contribution of the internal electronic structure of the ions embedded in the dense plasma is calculated using a scheme 27] which self-consistently: (i) builds separate models for different ionization stages in the plasma, taking into account the real structure of bound states (configurations, LS terms); (ii) uses Boltzmann statistics to sum up the internal partition functions of these ions; (iii) takes into account spreading of bound states into energy bands as they are pressure ionized; and (iv) treats quantum mechanically the free electron background around each ion thus resolving resonances. Points (i) and (ii) make our model different from average atom ones. The closest "ion-in-plasma" theoretical model is that of Pain and Blenski [24], where ions are treated separately (using superconfigurations), but screening is introduced through a Thomas-Fermi approach for the free electrons. The applicability of our model is tested by numerical calculations of thermodynamic functions, which we compare with the FGV models.

In Sec. III we present the free energy model. The technique for the calculation of thermodynamic functions at equilibrium is described in Sec. IIII In Sec. IV we discuss the results of the EOS calculations for carbon plasma, and in Sec. $\mathbb{\nabla}$ we give conclusions.

\section{FREE ENERGY MODEL}

Consider a plasma consisting of $N_{e}$ free electrons and $N_{i}$ heavy ions with numbers of bound electrons $\nu$ from 0 to $Z_{\text {nuc }}$ (where $Z_{\text {nuc }}$ is the element charge number) in a volume $V$. Let us write the total Helmholtz free energy as $F_{\text {tot }}=F_{e}+F_{i}+F_{\text {ex }}$, where $F_{i, e}$ denote the ideal free energy of ions and free electrons, respectively, and $F_{\mathrm{ex}}$ is the excess (nonideal) part, which arises from interactions. $F_{i}$ is the free energy of an ideal Boltzmann gas mixture, which can be written as $F_{i}=N_{i} k_{\mathrm{B}} T\left[\ln \left(n_{i} \lambda_{i}^{3}\right)-1\right]-S_{\mathrm{mix}} T$, where $\lambda_{i}=$ $\left(2 \pi \hbar^{2} / m_{i} k_{\mathrm{B}} T\right)^{1 / 2}$ is the thermal wavelength of the ions, $m_{i}$ is the ion mass, $S_{\text {mix }}=-N_{i} k_{\mathrm{B}} \sum_{\nu} x_{\nu} \ln x_{\nu}$ is the entropy of mixing, and $x_{\nu}=N_{\nu} / N_{i}$ is the number fraction of the ions of the $\nu$-th type $\left(\sum_{\nu} x_{\nu}=1\right)$. For the electrons at arbitrary degeneracy, $F_{e}$ can be expressed through Fermi-Dirac integrals and approximated by an- alytical formulae 25]. The main complication is the calculation of the nonideal term, which is quite nontrivial at high densities. It includes a contribution due to the building of localized bound states of the ions, and the long range Coulomb interactions between these ions and free electrons. We write

$$
F_{\text {ex }}=F_{e e}+F_{i i}+F_{i e}+F_{\text {int }},
$$

where the first three terms represent the contributions of electron-electron, ion-ion, and ion-electron interactions, respectively, and $F_{\text {int }}$ is the contribution due to the internal degrees of freedom of the ions, that involves sums over bound states. Equation (11) does not imply a strict separation of the terms on its right-hand side: No strict definition of free and bound electrons nor ions exists in a dense plasma. In general, the terms must be interdependent and evolve in a correlated way. Our approach to this difficulty consists in calculating self-consistent models for the ions embedded in the plasma, coupling them with a model for the long range interaction, and minimizing the resulting total free energy $F_{\text {tot }}$.

\section{A. Free energy of a fully ionized plasma}

A fully ionized electron-ion plasma which contains only one ion species is characterized by three parameters: the ion charge $Z e$, the electron density parameter $r_{s}$, and the ion Coulomb coupling parameter $\Gamma$ :

$$
r_{s}=\frac{a_{e}}{a_{0}}, \quad \Gamma=\left(\frac{4 \pi n_{e}}{3}\right)^{1 / 3} \frac{Z^{5 / 3} e^{2}}{k_{\mathrm{B}} T},
$$

where $n_{e}$ is the electron number density and $a_{0}=$ $\hbar^{2} / m_{e} e^{2}$ is the Bohr radius. The Helmholtz free energy of the fully ionized plasma is described by analytical fitting formulae 25, 26], which are applicable at high densities $\left(r_{s} \lesssim 1\right.$, arbitrary $\left.\Gamma\right)$ or high temperatures (small $\Gamma$ ). When neither $r_{s}$ nor $\Gamma$ are small, the plasma cannot be considered as fully ionized.

In a multicomponent fully ionized, dense plasma with different ion charges $Z_{\nu} e$, the "linear mixing rule" has been shown to be very accurate [28, 29, 30]:

$$
F_{\mathrm{ex}}^{\mathrm{fi}}\left(N_{i}, V, T,\left\{x_{\nu}\right\},\left\{Z_{\nu}\right\}\right)=N_{i} k_{\mathrm{B}} T \sum_{\nu} x_{\nu} f_{\nu},
$$

where

$$
f_{\nu}=f_{\mathrm{ex}}^{\mathrm{fi}}\left(n_{e}, T, Z_{\nu}\right)=\left.F_{\mathrm{ex}}^{\mathrm{fi}}\right|_{x_{\nu}=1} / N_{i} k_{\mathrm{B}} T
$$

is obtained from $F_{\mathrm{ex}}^{\mathrm{fi}}=F_{e e}+F_{i i}+F_{i e}$ (the superscript "fi" indicates full ionization). In Eq. (4) $n_{e}$ takes the value implied by the electroneutrality: $n_{e}=n_{i} \bar{Z}$, where $n_{i}=$ $N_{i} / V$ is the ion number density, and $\bar{Z} e=\sum_{\nu} x_{\nu} Z_{\nu} e$ is the mean ion charge. An effective ion Coulomb parameter for a multicomponent plasma is obtained by replacing $Z^{5 / 3}$ with $\sum_{\nu} x_{\nu} Z_{\nu}^{5 / 3}$ in Eq. (2) for $\Gamma$. 


\section{B. Bound-state contribution to the free energy}

In order to evaluate $F_{\text {int }}$, we calculate the ionic structure in the plasma using the scheme described in [27]. It is based on the ion-sphere approximation, which replaces the actual plasma environment for every ion by the statistically averaged boundary conditions for the electron wave functions within a spherical volume centered at the ionic nucleus. At present we do not include neutral atoms $\left(\nu=Z_{\text {nuc }}\right)$, which is justified at the temperatures and densities where the ionization degree of the plasma is high. For each ion containing $\nu$ bound electrons, a radius of the ion sphere $R_{\nu}$ is determined self-consistently from the requirement that the sphere is overall electrically neutral. The Hamiltonian for the ion $\nu$ is written as $H_{\nu}=\sum_{i=1}^{\nu} h_{\nu}\left(\boldsymbol{r}_{i}\right)+W_{\nu}$, where

$$
\begin{aligned}
& h_{\nu}(\boldsymbol{r})=-\frac{\hbar^{2}}{2} \nabla^{2}+V_{\mathrm{at}}^{\nu}(r)+V_{\mathrm{f}}^{\nu}(r), \\
& W_{\nu}=\sum_{i=1}^{\nu}\left(-\frac{Z_{\mathrm{nuc}} e^{2}}{r_{i}}-V_{\mathrm{at}}^{\nu}\left(r_{i}\right)\right)+\sum_{i<j}^{\nu} \frac{e^{2}}{\left|\boldsymbol{r}_{i}-\boldsymbol{r}_{j}\right|},
\end{aligned}
$$

$V_{\mathrm{f}}^{\nu}$ is the potential due to the plasma on the ion $\nu$, that must be determined self-consistently, $W_{\nu}$ is responsible for the $L S$ splitting of spectroscopic terms, and $V_{\text {at }}^{\nu}$ is a scaled Thomas-Fermi potential of the nucleus and $\nu-1$ bound electrons 31]. Note that $V_{\text {at }}^{\nu}$ disappears in $H_{\nu}$. It is used to build an effective one-electron Hamiltonian $h_{\nu}$, which generates a one-electron wave functions basis. The coordinate parts $\psi_{n l m}^{\nu}$ of these functions are obtained from the Schrödinger equation

$$
h_{\nu} \psi_{n l m}^{\nu}=\epsilon_{\nu n l} \psi_{n l m}^{\nu}(\boldsymbol{r}) .
$$

Then $H_{\nu}$ is diagonalized in a subspace of Slater determinants generated by a set of $\psi_{n l m}^{\nu}$. The $\nu$-electron energies of the bound states are well approximated as $E_{\nu \alpha}=E_{\nu \alpha}^{0}+\sum_{(n l) \in \alpha}\left(\epsilon_{\nu n l}-\epsilon_{\nu n l}^{0}\right)$, where $E_{\nu \alpha}^{0}$ and $\epsilon_{\nu n l}^{0}$ are calculated for the isolated ion, and $\alpha=$ $(n l)_{1}(n l)_{2} \ldots(n l)_{\nu}{ }^{2 S+1} L$ defines a particular $L S$ term of a configuration. The separation of $H_{\nu}$ into parts (5) and (6) allows one to capture the plasma effects in oneelectron energies and wave functions through Eq. (5), while the $\nu$-electron structure is retained through the contribution $W_{\nu}$. The boundary condition at $R_{\nu}$ for Eq. (7) does not noticeably affect $E_{\nu \alpha}$ except near the densities where the corresponding term $\alpha$ becomes pressureionized. The latter case will be addressed below.

The free electron density $n_{\mathrm{f}}(r)$ and the potential $V_{\mathrm{f}}^{\nu}(r)$ are determined self-consistently, using the local density approximation of the density functional theory. The oneelectron wave functions $\psi_{\epsilon l m}^{\nu}(\boldsymbol{r})$ of the partial scattering waves are calculated from the Schrödinger equation

$$
\begin{aligned}
(- & \frac{\hbar^{2}}{2} \nabla^{2}-\frac{Z_{\mathrm{nuc}} e^{2}}{r}+V_{\mathrm{b}}^{\nu}(r) \\
& \left.+V_{\mathrm{f}}^{\nu}(r)+V_{\mathrm{xc}}^{\nu}(r)\right) \psi_{\epsilon l m}^{\nu}(\boldsymbol{r})=\epsilon \psi_{\epsilon l m}^{\nu}(\boldsymbol{r}) .
\end{aligned}
$$

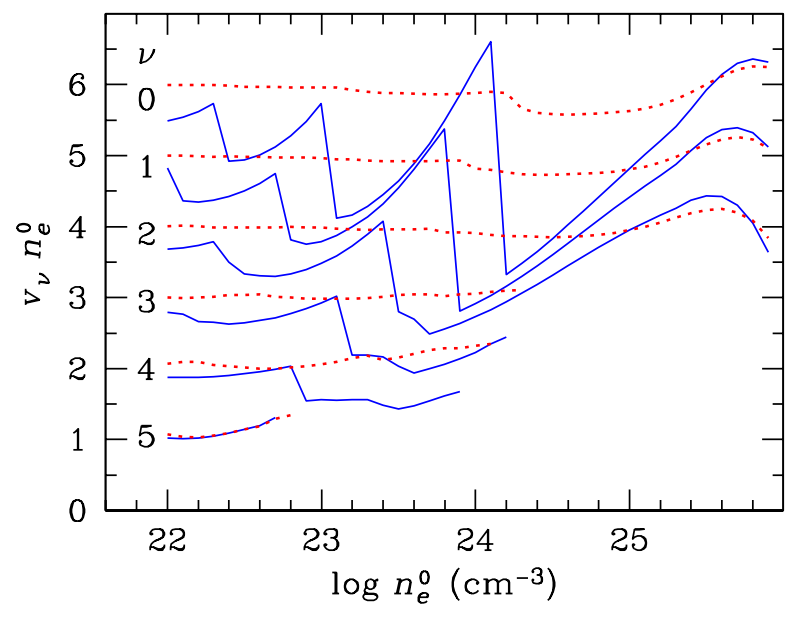

FIG. 1: Neutrality volumes of carbon ions, $v_{\nu}$ normalized to the fiducial number density of free electrons $n_{e}^{0}$ (see text), as functions of $n_{e}^{0}$ for $T=2.32 \times 10^{5} \mathrm{~K}$ (solid lines) and $T=3.71 \times 10^{6} \mathrm{~K}$ (dotted lines) for carbon ions with $\nu$ bound electrons $(\nu=0,1, \ldots, 5)$. The curves for the three lowest degrees of ionization $(\nu=3,4,5)$ end at the $n_{e}^{0}$ values at which there remain no relevant bound states $\left(\epsilon_{-}\right.$become positive for $2 s$ state in $1 s^{2} 2 s$ and $1 s^{2} 2 s^{2}$, and $2 p$ state in $\left.1 s^{2} 2 s^{2} 2 p\right)$.

Here, $V_{\mathrm{xc}}^{\nu}$ is the exchange-correlation potential [32], $V_{\mathrm{b}}^{\nu}$ and $V_{\mathrm{f}}^{\nu}$ are obtained from the Poisson equation: $\nabla^{2} V_{\mathrm{b}}^{\nu}=$ $-4 \pi n_{\mathrm{b}}^{\nu} e^{2}, \nabla^{2} V_{\mathrm{f}}^{\nu}=-4 \pi n_{\mathrm{f}}^{\nu} e^{2}$, and the number densities $n_{\mathrm{b}}^{\nu}(r)$ and $n_{\mathrm{f}}^{\nu}(r)$ are calculated as the squared moduli of the wave functions for the bound and free electrons, respectively, summed with the statistical weights appropriate for a given $T$. For the bound electrons, these weights are proportional to $w_{\nu \alpha} d_{\nu \alpha} \exp \left(-E_{\nu \alpha} / k_{\mathrm{B}} T\right)$, where $d_{\nu \alpha}=(2 S+1)(2 L+1)$ is the level degeneracy, and $w_{\nu \alpha}$ is an occupation probability defined below. The density of states per unit volume for the $l$ th partial wave of the free electrons at a given energy $g_{\nu l}(\epsilon)$ is determined with account of the contribution from resonances (Friedel terms; see Ref. 27] for details). The energy distribution of the free electrons is assumed $\propto g_{\nu l}(\epsilon) /\left(1+\exp \left[\left(\epsilon-\mu_{e}\right) / k_{\mathrm{B}} T\right]\right)$. The free parameters of the model are $T$ and the electron chemical potential $\mu_{e}$. In thermodynamic equilibrium, $\mu_{e}$ is the same for all ions, but as different ionization stages have different neutrality sphere radii as well as different numbers of neutralizing free electrons inside them, $\mu_{e}$ can be related to the mean free electron density only after the global free-energy minimization (Sec. III) has given the relative populations of the ions.

The neutrality of the ion sphere is ensured by the selfconsistent determination of $R_{\nu}$ such that

$$
\frac{\mathrm{d}}{\mathrm{d} r}\left[-\frac{Z_{\mathrm{nuc}}}{r}+V_{\mathrm{b}}^{\nu}(r)+V_{\mathrm{f}}^{\nu}(r)\right]_{r=R_{\nu}}=0 .
$$

Associated with this radius is the neutrality volume $v_{\nu}=4 \pi R_{\nu}^{3} / 3$. In the model of a uniform electron background, that neglects the interactions of free electrons 
with ions, one has $v_{\nu}=v_{\nu}^{0}=\left(Z_{\text {nuc }}-\nu\right) / n_{e}^{0}$, where $n_{e}^{0}\left(\mu_{e}, T\right)=V^{-1} \partial F_{e} /\left.\partial \mu_{e}\right|_{V, T}$ is the number density of free electrons in the uniform gas model. With allowance for interactions of the free electrons with the ions and bound electrons, $v_{\nu}$ deviates from $v_{\nu}^{0}$, as illustrated in Fig. 11 The drops of the plotted curves at certain densities, which are especially sharp at the lower temperature, are the consequence of pressure ionization of separate levels: when a $n l$ level of ion $\nu$ crosses the continuum limit and appears as a resonance in the neighboring ionization state $\nu-1$, the latter ion sphere shrinks to compensate this increase in the free electron density of states.

With increasing $\mu_{e}$ (or $n_{e}^{0}$ ), the radius $R_{\nu}$ decreases, the wave functions $\psi_{n l m}^{\nu}(r)$ become distorted, and the energies $\epsilon_{\nu n l}$ spread into a band. We estimate a band width by solving Eq. (7) with two alternative boundary conditions: either $\psi_{n l m}^{\nu}\left(R_{\nu}\right)=0$, or $\partial \psi_{n l m}^{\nu} / \partial r=0$ at $r=R_{\nu}$. These two conditions give two energies which we interpret as the upper $\left(\epsilon_{+}\right)$and lower $\left(\epsilon_{-}\right)$edges of the band (Fig. 21). Eventually $\epsilon_{+}$becomes positive. We interpret the electrons with $0<\epsilon<\epsilon_{+}$as quasifree and exclude them from the internal partition function of the ion. We introduce an occupation probability $w_{\nu n l}$, equal to the statistical weight of electrons with $\epsilon<0$ (the significance and thermodynamic meaning of occupation probabilities in the chemical picture of plasmas has been discussed, e.g., in 20, 33, 34]). Assuming for the bands the Hubbard [35] density of states

$g(\epsilon)=\frac{2}{\pi \delta^{2}} \sqrt{\delta^{2}-(\epsilon-\bar{\epsilon})^{2}}, \quad \delta=\frac{\epsilon_{+}-\epsilon_{-}}{2}, \quad \bar{\epsilon}=\frac{\epsilon_{+}+\epsilon_{-}}{2}$,

we obtain, for $\epsilon_{-}<0<\epsilon_{+}$,

$$
\begin{gathered}
w_{\nu n l}=\frac{1}{2}-\frac{y}{\pi} \sqrt{1-y^{2}}-\frac{1}{\pi} \arcsin (y), \\
y=\left(\epsilon_{+}+\epsilon_{-}\right) /\left(\epsilon_{+}-\epsilon_{-}\right) .
\end{gathered}
$$

The occupation probability of a term $\alpha$ is $w_{\nu \alpha}=$ $\prod_{(n l) \in \alpha} w_{\nu n l}$. For all electron shells $n l$, except the $K$ shell, $\epsilon_{-}$becomes positive at sufficiently high $\mu_{e}$; in this case $w_{\nu n l}=0$ and the bound state disappears. The lowest curves in Fig. 10nd at the densities where the bound states cease to exist in the plasma for a given $\nu$.

For the $K$ shell of $\mathrm{H}$ - and He-like ions, $\epsilon_{-}$is negative at any density. Asymptotically, at large $\mu_{e}$ (small $R_{\nu}$ ) and for a uniform density $n_{e}, \epsilon_{-} \simeq-0.3\left(Z_{\text {nuc }}-\nu\right) e^{2} / R_{\nu}$, while $\epsilon_{+} \simeq 0.5 m_{e}^{-1}\left(\pi \hbar / R_{\nu}\right)^{2}$. Therefore, at high densities $w_{\nu n l} \simeq\left(4 \sqrt{6} / \pi^{4}\right)\left[\left(Z_{\mathrm{nuc}}-\nu\right) R_{\nu} / 5 a_{0}\right]^{3 / 2} \propto n_{e}^{-1 / 2}$.

The electrons, that populate the bands overlapping with the continuum, are delocalized, and thus add to the free-electron degeneracy through the Pauli exclusion principle. It means that at a given $n_{i}$ the presence of such electrons increases $\mu_{e}$. Conversely, at a fixed $\mu_{e}$, the electrons that are pushed into continuum have a larger neutrality volume, corresponding to the unscreened shell. Since the share of these quasifree electrons is $\left(1-w_{\nu \alpha}\right)$, an effective ion charge for such a partially delocalized state [to be used in Eq. (4)] is $Z_{\nu}=Z_{\text {nuc }}-\nu w_{\nu}-\nu^{*}\left(1-w_{\nu}\right)$,

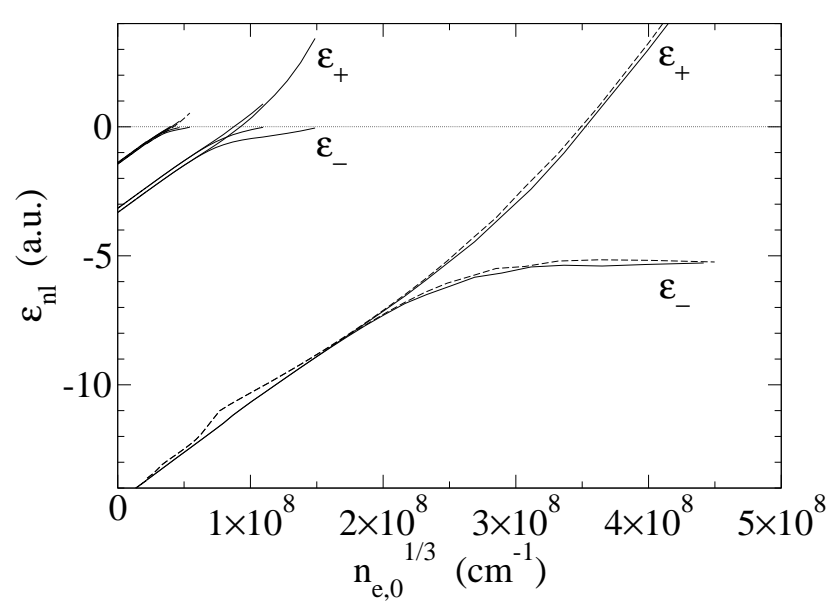

FIG. 2: Monoelectronic energies of He-like carbon $(\nu=2)$ as a function of the fiducial electronic density $n_{e}^{0}$; solid line: for $T=3.71 \times 10^{6} \mathrm{~K}$ and $1 s, 2 s, 2 p, 3 s, 3 p, 3 d$ states (from bottom to top); dashed line: for $T=2.32 \times 10^{5} \mathrm{~K}$ and the $1 \mathrm{~s}$ state. Upper and lower limits of energy bands are marked as $\epsilon_{+}$and $\epsilon_{-}$.

where $w_{\nu}=w_{\nu \alpha}$ for the lowest level $\alpha$ of the ion with $\nu$ electrons, and $\nu^{*}<\nu$ is the number of electrons remaining on the inner shells $\left(\nu^{*}=0\right.$ for $1 s$ and $\left.1 s^{2}\right)$.

The contribution of the internal degrees of freedom into the free energy is calculated as $F_{\text {int }}=-\sum_{\nu} N_{\nu} k_{\mathrm{B}} T \ln \mathcal{Z}_{\nu}$, where $\mathcal{Z}_{\nu}=\sum_{\alpha} w_{\nu \alpha} d_{\nu \alpha} \exp \left(-E_{\nu \alpha} / k_{\mathrm{B}} T\right)$ is the internal partition function of the ion in the plasma.

\section{Total free energy model}

We evaluate $F_{e e}+F_{i i}+F_{i e}$ in Eq. (11) as described in Sec. IA Albeit this is not strictly correct for ions with bound states, which are not pointlike, we need this approximation to make practical EOS calculations.

The total free energy, normalized to $N_{i} k_{\mathrm{B}} T$, can be written as

$$
\begin{aligned}
& f \equiv \frac{F_{\text {tot }}}{N_{i} k_{\mathrm{B}} T}=f^{\mathrm{fi}}+f_{\text {int }}, \\
& \text { where } \quad f^{\mathrm{fi}}=\sum_{\nu} x_{\nu} f_{\nu}+f_{i}+\bar{Z} f_{e}, \\
& f_{i}=\ln \left(n_{i} \lambda_{i}^{3}\right)-1-s_{\text {mix }}, \\
& f_{e}=\chi_{e}-p_{e}, \quad \chi_{e}=\frac{\mu_{e}}{k_{\mathrm{B}} T}, \quad p_{e}=\frac{P_{e}}{n_{e} k_{\mathrm{B}} T}, \\
& f_{\text {int }}=\frac{F_{\text {int }}}{N_{i} k_{\mathrm{B}} T}, \quad s_{\text {mix }}=\frac{S_{\text {mix }}}{N_{i} k_{\mathrm{B}}},
\end{aligned}
$$

and $P_{e}$ is the free-electron pressure. All terms of $f^{\text {fi }}$ can be calculated using the fitting formulae [25, 26], and only $f_{\text {int }}$ should be evaluated numerically. 


\section{THERMODYNAMIC EQUILIBRIUM}

\section{A. Equilibrium conditions}

Thermodynamic equilibrium at constant $V$ and $T$ realizes at the minimum of the Helmholtz free energy $F_{\text {tot }}$. Since the total number of the ions in all ionization states is fixed, this minimum must be found under the constraint $\sum_{\nu} N_{\nu}=N_{i}$. The charge neutrality condition is satisfied automatically, because the total number of electrons equals $Z_{\text {nuc }}$ in each ion cell by construction, however at cost of the a priori unconstrained volume. In order to maintain $V=$ constant, one should impose the condition $\sum_{\nu} N_{\nu} v_{\nu}=V$.

These equilibrium conditions can be written as

$$
\begin{aligned}
& f=\text { minimum; } \quad G_{N}=G_{V}=1 ; \quad x_{\nu} \geq 0, \forall \nu \\
& G_{N}=\sum_{\nu} x_{\nu}, \quad G_{V}=n_{i} \sum_{\nu} x_{\nu} v_{\nu}
\end{aligned}
$$

where $f=f\left(\chi_{e}, T,\left\{x_{\nu}\right\}\right)$ is given by Eq. (12), $n_{i}$ and $T$ are fixed, while $\chi_{e}$ and $x_{\nu}$ may vary.

\section{B. Finding the equilibrium}

While solving the constrained minimization problem (13), we take into account the condition $G_{N}=1$ explicitly, by setting $x_{0}=1-\sum_{\nu=1}^{Z_{\text {nuc }}-1} x_{\nu}$, and discard those $\left\{x_{\nu}\right\}$ sets which would result in the negative right-hand side of this equation. As mentioned above, we do not consider the neutral atoms $\left(\nu=Z_{\text {nuc }}\right)$. In order to satisfy the constraint $G_{V}=1$, we use the Lagrange multiplier method. Namely, we minimize an auxiliary function

$$
\Phi\left(n_{i}, T ; \chi_{e},\left\{x_{\nu}\right\} ; \lambda\right)=f-\lambda G_{V}+\lambda^{2}\left(G_{V}-1\right)^{2}
$$

with respect to its arguments $\chi_{e}$ and $x_{\nu}(1 \leq \nu \leq$ $\left.Z_{\text {nuc }}-1\right)$ for different values of the Lagrange multiplier $\lambda$, and find the $\lambda$ value that gives $G_{V}=1$ at the minimum. The last (quadratic) term in Eq. (14) is an empirical regularization term which accelerates the solution. The solution provides the equilibrium values of $F_{\text {tot }}, \chi_{e}$, $x_{\nu}$, and $n_{e}=\bar{Z} n_{i}$.

At each value of $\lambda$, we approach min $\Phi$ in two stages: first, a rough position of the minimum is found by the simplex method, and then it is refined by the Powell's conjugate-direction procedure [36]. In order to filterout false local minima, the minimization procedure is repeated several times with different initial sets of parameters, and the absolute minimum is selected. A search for the root of the equation $G_{V}(\lambda)=1$ is performed by bracketing and bisection, Because of the complicated dependence of $f$ and $G_{V}$ on the set of $x_{\nu}$, and due to the limited accuracy of minimization, $G_{V}(\lambda)$ may exhibit a numerical discontinuity, which sometimes disallows the bisection to converge, so we have tried several initial guesses of $\lambda$ in such cases.

\section{Calculating thermodynamic functions}

Once $F_{\text {tot }}$ is calculated for a range of temperatures and densities, all thermodynamic functions can be found from its derivatives. The first derivatives give the pressure $P=-\partial F_{\text {tot }} /\left.\partial V\right|_{T}$, entropy $S=-\partial F_{\text {tot }} /\left.\partial T\right|_{V}$, and internal energy $U=-T^{2} \partial /\left.\partial T\left(F_{\text {tot }} / T\right)\right|_{V}=F+T S$. The second derivatives give, for example, specific heat $C_{V}=$ $\partial U /\left.\partial T\right|_{V}$ and the pressure exponents (temperature and density logarithmic derivatives) $\chi_{T}=\partial \ln P /\left.\partial \ln T\right|_{V}$ and $\chi_{\rho}=-\partial \ln P /\left.\partial \ln V\right|_{T}$. In these derivatives, $N_{i}$ is kept fixed, but $x_{\nu}$ depend on $V$ and $T$, following the solution in Sec.IIIB

Although such calculation looks simple, it is technically complicated. We achieved the accuracy of $f$ within 0.003 over the $\rho-T$ domain where the electron degeneracy is weak or moderate $\left(\chi_{e} \lesssim 10\right)$, and to four digits in the strongly degenerate regime (where $\chi_{e} \gg 1$ ), but this is insufficient for an accurate evaluation of the second and mixed derivatives of $f$. The difficulty is partly overcome by filtering the calculated values. We performed calculations on a grid of $(\rho, T)$ points and evaluated the derivatives at each $(\rho, T)$ point from using the least-squares fit to the $F$ values at a hundred of neighboring grid points.

This filtering is not sufficient, if the electrons are strongly degenerate. In this case, the $T$-derivatives of $\ln F_{\text {tot }}, \ln U$, and $\ln P$ are so small that a tiny numerical noise may preclude their evaluation. Fortunately, in this regime these derivatives are mainly determined by $f^{\mathrm{fi}}$. We use the following modification of Eq. (12a):

$$
f=f^{\mathrm{fi}}+s_{\text {mix }}+f^{\prime}, \quad f^{\prime}=\sum_{\nu} x_{\nu} \ln \left(x_{\nu} / \mathcal{Z}_{\nu}\right) .
$$

The values of $f^{\prime}, x_{\nu}$, and $\bar{Z}$, and their $\rho$ - and $T$ derivatives are calculated numerically, as described above, whereas $f^{\text {fi }}$ and its derivatives are obtained from the analytical fits [25, 26].

The calculated functions $C_{V}, \chi_{T}$, and $\chi_{\rho}$ still exhibit a considerable numerical noise. To suppress it, we again employ the least-squares filtering. Improved values of pressure, consistent with the filtered $\chi_{T}$, are obtained by numerical integration of the equation $\ln P=\int \chi_{T} \mathrm{~d} t$, starting from the lowest isotherm.

The thermodynamic stability $\left(C_{V}>0, P>0\right)$ and normality $\left(\chi_{T}>0\right)$ require that $S / N_{i} k_{\mathrm{B}}$ monotonically increases with decreasing $\rho$ or increasing $T$. To maintain these properties, we calculate $S$ by integration of the equations $\partial S /\left.\partial V\right|_{T}=\chi_{T} P / T$ and $\partial S /\left.\partial T\right|_{V}=C_{V} / T$, starting from the highest $\rho$ and lowest $T$.

\section{RESULTS FOR CARBON}

We have calculated the EOS for carbon at $2.34 \times$ $10^{5} \mathrm{~K} \leq T \leq 3.63 \times 10^{6} \mathrm{~K}$ and $10^{-4} \mathrm{~g} \mathrm{~cm}^{-3} \leq \rho \leq 10^{4}$ $\mathrm{g} \mathrm{cm}^{-3}$; at still higher $\rho$ the carbon plasma is fully ionized. For lower temperatures, $7.5 \times 10^{4} \mathrm{~K} \leq T \leq 2.34 \times$ 


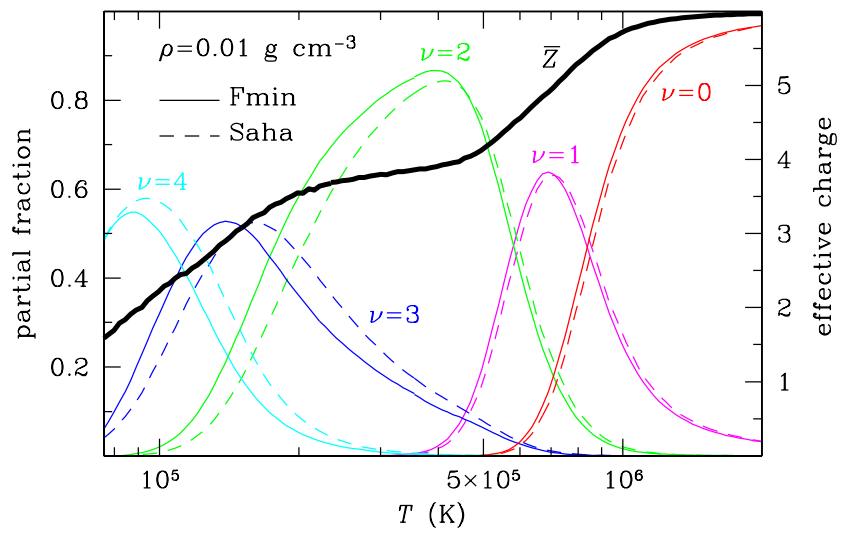

FIG. 3: Number fractions of different carbon ions in the plasma (left vertical axis) versus temperature at $\rho=10^{-2}$ $\mathrm{g} \mathrm{cm}^{-3}$. Solid lines: accurate results; dashed lines: Saha approximation with current partition functions. Numbers of bound electrons $\nu$ are marked near the curves. The thick solid curve shows the mean effective charge $\bar{Z}$ (right axis).

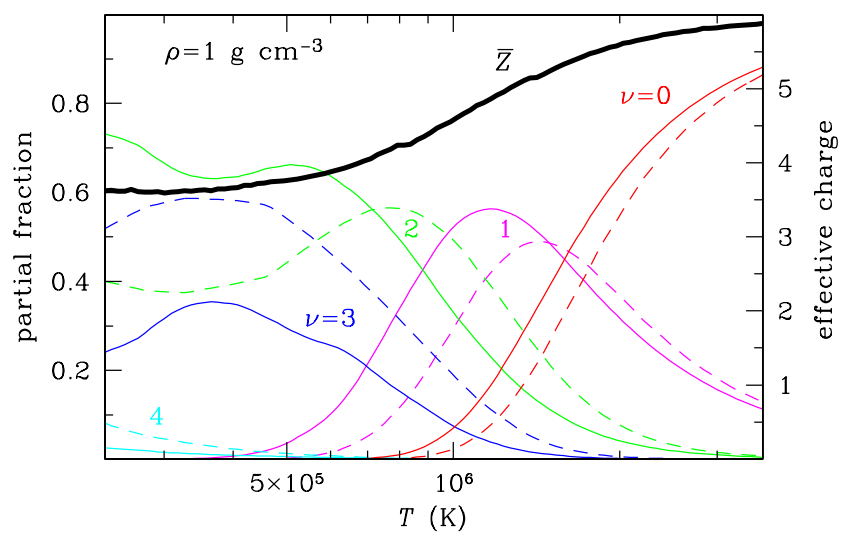

FIG. 4: The same as in Fig. 3 but at $\rho=1 \mathrm{~g} \mathrm{~cm}^{-3}$.

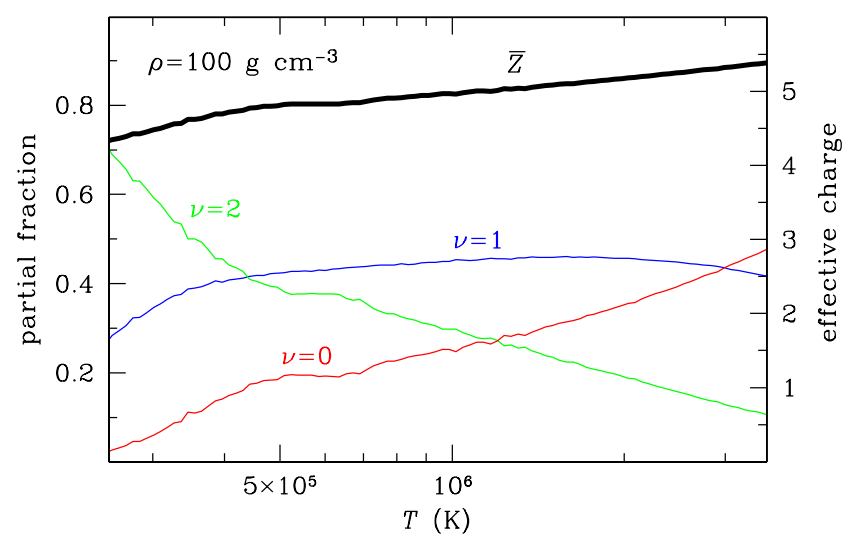

FIG. 5: Number fractions (left axis) and the mean effective charge $\bar{Z}$ (right axis) versus temperature at $\rho=100 \mathrm{~g} \mathrm{~cm}^{-3}$.

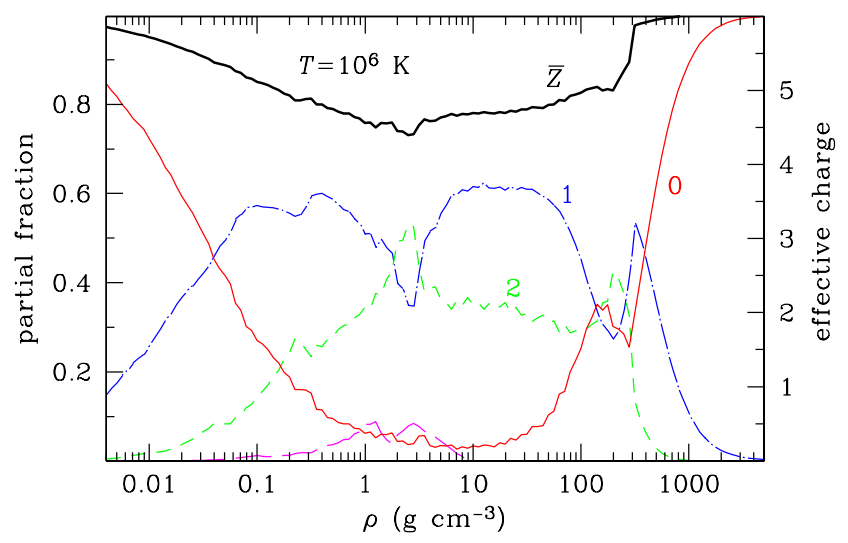

FIG. 6: Same as in Fig. 5 but versus density at constant $T=10^{6} \mathrm{~K}$.

$10^{5} \mathrm{~K}$, we have calculated the EOS at $10^{-4} \mathrm{~g} \mathrm{~cm}^{-3} \leq$ $\rho \leq 0.04 \mathrm{~g} \mathrm{~cm}^{-3}$.

Figures 315 show the $T$-dependences of ion number fractions $x_{\nu}$ and the mean effective charge $\bar{Z}$. At the lower densities, the electrons are nondegenerate. In this case, the mean ionization degree and $\bar{Z}$ depend sensitively on temperature. At the high density (Fig. [5), the electron degeneracy is significant, and the number of free electrons is mainly controlled by pressure, rather than temperature, so that $\bar{Z}$ varies weakly. However, the state of the bound electrons still depends appreciably on $T$ : most of them are in the $1 s$ state at the higher $T$ and in the $1 s^{2}$ state at the lower $T$.

The nonideality effects are less important at lower density. Therefore, the abundance of individual ion species at low densities can be evaluated from the Saha equation

$$
\frac{x_{\nu+1}}{x_{\nu}}=\frac{\mathcal{Z}_{\nu+1}}{\mathcal{Z}_{\nu}} \frac{n_{e}}{2}\left(\frac{2 \pi \hbar^{2}}{m_{e} k_{\mathrm{B}} T}\right)^{3 / 2},
$$

as illustrated by Fig. [3 for $\rho=0.01 \mathrm{~g} \mathrm{~cm}^{-3}$. Note that the shifts of bound-state levels in the plasma environment are included in $\mathcal{Z}_{\nu}$. At $\rho \ll 0.01 \mathrm{~g} \mathrm{~cm}^{-3}$, this approximation and our calculations give identical results (this is one of the checks of our calculations), but at $\rho \gtrsim 0.01 \mathrm{~g} \mathrm{~cm}^{-3}$, Eq. (16) becomes progressively inaccurate (Fig. 4). The differences between Saha and our models in Figs. 3] and 4 are due to the configurational effects (i.e., the deviations of the neutrality volumes from their ideal values; see Fig. 1) and the Coulomb plasma nonideality (Sec. IIA).

The $\rho$ dependences of the ionization states at $T=$ $10^{6} \mathrm{~K}$ are shown in Fig. 6] They exhibit pronounced maxima and minima due to the pressure ionization of particular bound states in particular ionization stages. These features are related to swelling and shrinking of the individual neutrality volumes relative to their rigid-background values (Fig. 1) and the corresponding changes in the internal partition functions.

Figure 7 presents normalized pressure as a function of density at $T=5 \times 10^{5} \mathrm{~K}$. The vertical scale is smaller for 


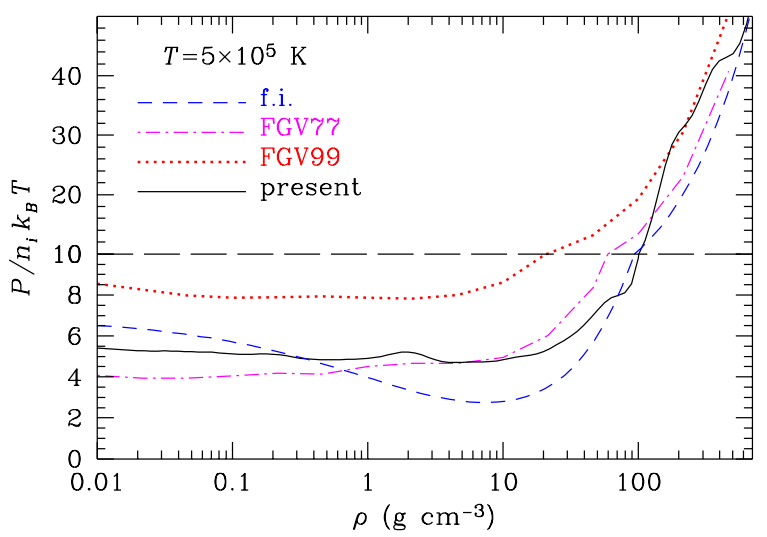

FIG. 7: Isotherms of normalized pressure $P / n_{i} k_{\mathrm{B}} T$ for $T=$ $5 \times 10^{5} \mathrm{~K}$. The present data (solid lines) are compared with the FGV77 (dot-dashed lines), FGV99 (dotted lines), and fully ionized plasma (dashed lines) models. Note the different scale in the figure above and below the horizontal long-dash line.

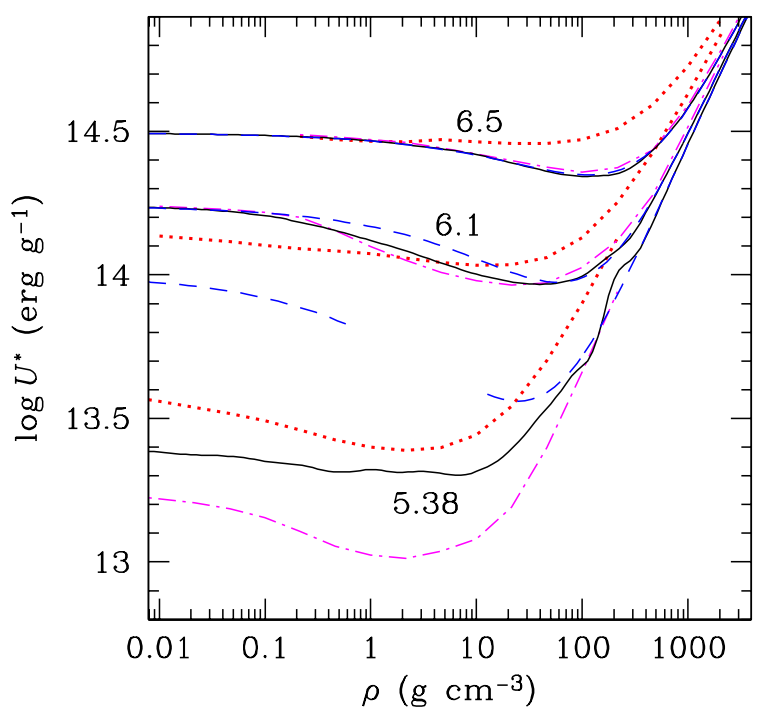

FIG. 8: Isotherms of internal energy for $T=2.4 \times 10^{5} \mathrm{~K}$, $1.26 \times 10^{6} \mathrm{~K}$, and $3.16 \times 10^{6} \mathrm{~K}$ (the curves are marked by $\log T$ values). The present data (solid lines) are compared with the FGV77 (dot-dashed lines), FGV99 (dotted lines), and fully ionized plasma (dashed lines) models.

the upper part of the figure, to take account of the rapidly growing pressure of degenerate electrons. The difference between our results and the FGV99 tables is in general of the same magnitude as the difference between FGV77 and FGV99. However, our isotherms exhibit more features. The slope of each isotherm varies near the densities where the ion composition of the plasma rapidly changes. These variations could not be revealed by the Thomas-Fermi model, but are easily grasped within the free-energy minimization technique. Related variations are seen in Fig. 8, which shows isotherms of the internal energy per unit mass, $U^{*}=\left(U+U_{0}\right) / N_{i} m_{i}$ measured from the energy level $-U_{0}$ of a nonionized ground-state

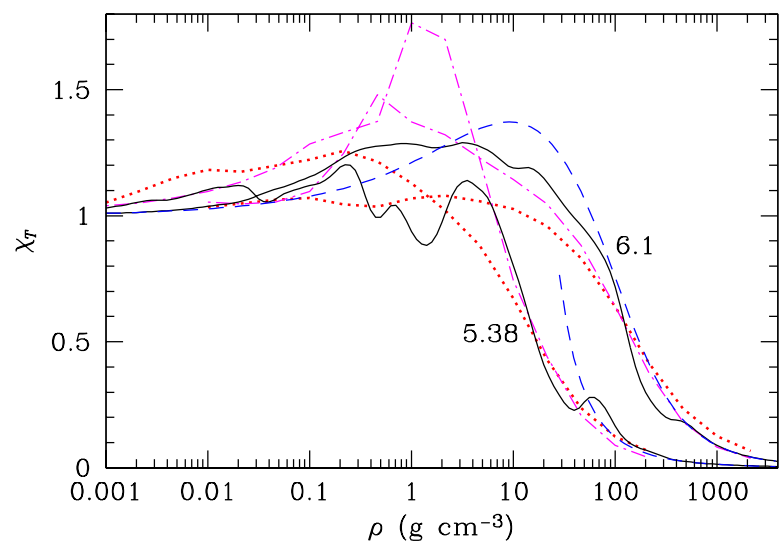

FIG. 9: Temperature exponent $\chi_{T}$ for isotherms $T=2.4 \times$ $10^{5} \mathrm{~K}$ and $1.26 \times 10^{6} \mathrm{~K}$, compared with the FGV77 (dotdashed lines), FGV99 (dotted lines), and fully ionized plasma (dashed lines) models (the curves marked with $\log T$ values).

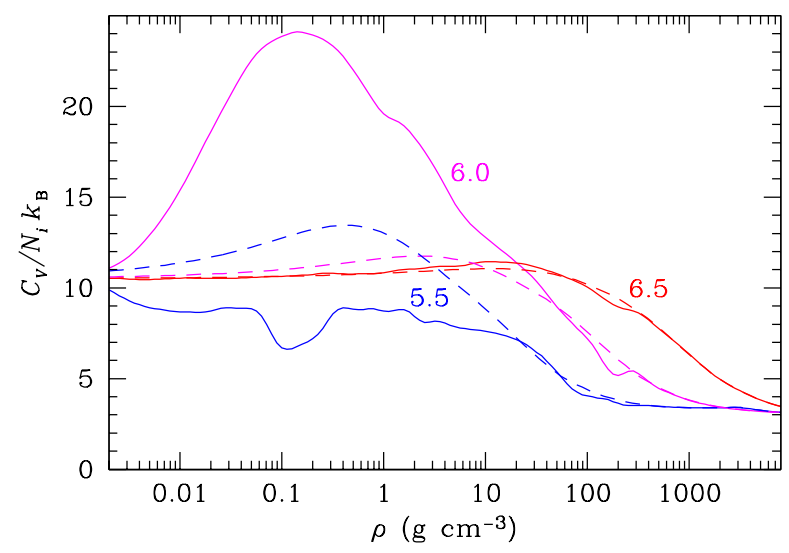

FIG. 10: Normalized specific heat at constant volume $c_{V}=$ $C_{V} / N_{i} k_{\mathrm{B}}$ for $T=3.16 \times 10^{5} \mathrm{~K}, 10^{6} \mathrm{~K}$, and $3.16 \times 10^{6} \mathrm{~K}$ (the curves marked with $\log T$ values). Present data (solid lines) are compared with the fully ionized plasma model (dashed lines).

carbon, which corresponds to a shift equal to $8.28 \times 10^{13}$ $\mathrm{erg}^{-1}$ with respect to the electron continuum level. The gap in the cold isotherm of the fully ionized plasma model (dashed line) corresponds to the region of instability of this model. Variations of the EOS due to the changing plasma composition with increasing density are also seen for the temperature derivative $\chi_{T}$, shown in Fig. 9

Figure [10] shows the normalized specific heat $c_{V} \equiv$ $C_{V} / N_{i} k_{\mathrm{B}}$ as a function of $\rho$ for three values of $T$. At low $\rho$, where the electrons are nondegenerate, the ideal-gas value of $c_{V}$ is $c_{V}^{\text {id }}=1.5(\bar{Z}+1)$. Actually $c_{V}$ approaches $c_{V}^{\text {id }}$ at $T \gtrsim 3 \times 10^{6} \mathrm{~K}$, where the ionization is almost complete but in general, these two quantities can differ considerably, in particular because the heat absorbed by a partially ionized medium is spent not only on the increase of the kinetic energy of the ions and electrons, but also on thermal ionization. At very high densities, the specific heat is mostly determined by the ions. For a classical ion 
solid $c_{V}=3$; for a strongly coupled ion liquid $c_{V} \approx 3.4$ near the melting $\Gamma$ (Eq. (17) of Ref. [26]). The corrections due to the ion-electron interactions and quantizing ion motions are within $10 \%$ in the high-density part of Fig. 10] This explains the limit $c_{V} \approx 3$ seen at high $\rho$.

At $\rho \gtrsim 1000 \mathrm{~g} \mathrm{~cm}^{-3}$, our model reproduces the EOS for a fully ionized nonideal plasma [26].

\section{CONCLUSIONS}

We have developed a model for calculation of the EOS for dense, partially ionized plasmas, based on the free energy minimization method and suitable for pressure ionization zones. The free energy model is constructed in the framework of the chemical picture of plasmas and includes detailed self-consistent accounting of quantum states of partially ionized atoms in the plasma environment. Occupation probability formalism based on the energy bands is used to treat pressure ionization.

The developed technique is applied to the carbon plasma at temperatures $T \gtrsim 10^{5} \mathrm{~K}$, which is relevant for inner envelopes of the carbon-rich white dwarfs or outer accreted envelopes of the neutron stars. For general astrophysical applications of various type of stars, it is desirable to extend the calculated EOS to other chemical elements, first of all to oxygen. We are planning to perform such calculations in near future.

\section{Acknowledgments}

The authors are grateful to G. Fontaine for providing updated tables of the FGV EOS. A.Y.P. acknowledges the hospitality of the theoretical astrophysics group at the Ecole Normale Supérieure de Lyon. The work of A.Y.P. and G.C. was partially supported by the CNRS French-Russian Grant No. PICS 3202. The work of A.Y.P. was also supported in part the RLSS Grant No. 1115.2003.2 and the RFBR Grant Nos. 05-02-16245, 0307-90200, and 05-02-22003.
[1] R. M. More, K. H. Warren, D. A. Young, and G. B. Zimmerman, Phys. Fluids 31, 3059 (1988).

[2] M. P. Surh, T. W. Barbee III, and L. H. Yang, Phys. Rev. Lett. 86, 5958 (2001).

[3] F. Perrot, M. W. C. Dharma-wardana, and J. Benage, Phys. Rev. E 65, 046414 (2002).

[4] C. Blancard and G. Faussurier, Phys. Rev. E 69, 016409 (2004).

[5] S. H. Glenzer, G. Gregori, R. W. Lee, F. J. Rogers, S. W. Pollaine, and O. L. Landen, Phys. Rev. Lett. 90, 175002 (2003).

[6] P. Renaudin, C. Blancard, J. Clerouin, G. Faussurier, P. Noiret, and V. Recoules, Phys. Rev. Lett. 91, 075002 (2003).

[7] D. Riley, I. Weaver, D. McSherry, M. Dunne, D. Neely, M. Notley, and E. Nardi, Phys. Rev. E 66, 046408 (2002).

[8] P. Audebert, R. Shepherd, K. B. Fournier, O. Peyrusse, D. Price, R. Lee, P. Springer, J.-C. Gauthier, and L. Klein, Phys. Rev. Lett. 89, 265001 (2002).

[9] D. Batani, A. Morelli, M. Tomasini, A. Benuzzi-Mounaix, F. Philippe, M. Koenig, B. Marchet, I. Masclet, M. Rabec, C. Reverdin, R. Cauble, P. Celliers, G. Collins, L. DaSilva, T. Hall, M. Moret, B. Sacchi, P. Baclet, and B. Cathala, Phys. Rev. Lett. 88, 235502 (2002).

[10] G. Fontaine and H. M. Van Horn, Astrophys. J. Suppl. Ser. 35, 293 (1976).

[11] F. D'Antona and I. Mazzitelli, Annu. Rev. Astron. Astrophys. 28, 139 (1990).

[12] G. M. Harris, J. E. Roberts and J. G. Trulio, Phys. Rev. 119, 1832 (1960).

[13] H. C. Graboske, Jr., D. J. Harwood, and F. J. Rogers, Phys. Rev. 186, 210 (1969).

[14] G., Fontaine, H. C. Graboske, Jr., and H. M. Van Horn, Astrophys. J. Suppl. Ser. 35, 293 (1977).

[15] D. Saumon and G. Chabrier, Phys. Rev. A 44, 5122 (1991); 46, 2084 (1992).

[16] J. M. Aparicio and G. Chabrier, Phys. Rev. E 50, 4948 (1994).

[17] C. Winisdoerffer and G. Chabrier, Phys. Rev. E 71,
$026402(2005)$

[18] D. Saumon, G. Chabrier, and H. M. Van Horn, Astrophys. J. Suppl. Ser. 99, 713 (1995).

[19] G. Fontaine (private communication, 1999).

[20] A. Y. Potekhin, Phys. Plasmas 3, 4156 (1996).

[21] F. J. Rogers, Phys. Plasmas, 7, 51 (2000).

[22] F.J. Rogers, F.J. Swenson, and C.A. Iglesias, Astrophys. J. 456, 902 (1996).

[23] V. Bezkrovniy, V. S. Filinov, D. Kremp, M. Bonitz, M. Schlanges, W. D. Kraeft, P. R. Levashov, and V. E. Fortov, Phys. Rev. E 70, 057401 (2004).

[24] J.-C. Pain and T. Blenski, J. Quant. Spectrosc. Radiat. Trans, 81, 355 (2003).

[25] G. Chabrier and A. Y. Potekhin, Phys. Rev. E 58, 4941 (1998).

[26] A. Y. Potekhin and G. Chabrier, Phys. Rev. E 62, 8554 (2000).

[27] G. Massacrier, J. Quant. Spectrosc. Radiat. Transfer 51, 221 (1994).

[28] J. P. Hansen, G. M. Torrie, and P. Vieillefosse, Phys. Rev. A 16, 2153 (1977).

[29] H. DeWitt, W. Slattery, and G. Chabrier, Physica B 228, 158 (1996).

[30] G. Chabrier and N. W. Ashcroft, Phys. Rev. A 42, 2284 (1990).

[31] W. Eissner and H. Nussbaumer, J. Phys. B 2, 1028 (1969).

[32] F. Perrot and M. W. C. Dharma-wardana, Phys. Rev. A 30, 2619 (1984).

[33] E. Fermi, Z. Phys. 26, 54 (1924).

[34] D. G.Hummer and D. Mihalas, Astrophys. J. 331, 794 (1988).

[35] J. Hubbard, Proc. R. Soc. London, Ser. A 281, 401 (1964).

[36] W. H. Press, S. A. Teukolsky, W. T. Vetterling, and B. P. Flannery, Numerical Recipes in Fortran, 2nd ed. (Cambridge University Press, Cambridge, UK, 1992). 